\title{
VrPET/CT: Development of a Rotating Multimodality Scanner for Small-Animal Imaging
}

\author{
Eduardo Lage, Juan José Vaquero, Senior Member, IEEE, Alejandro Sisniega, Samuel España, Gustavo Tapias, \\ Ángel Udías, Verónica García, Alexia Rodríguez-Ruano, Manuel Desco
}

\begin{abstract}
This work reports on the development and evaluation of the PET component of a PET/CT system for small-animal in-vivo imaging. The PET and CT subsystems are assembled in a rotary gantry in such a way that the center of rotation for both imaging modalities is mechanically aligned. The PET scanner configuration is based on 2 detector modules, each of which consist of 2 flat-panel type PS-PMTs (Hamamatsu, H8500) and $2(30 \times 30$ elements) LYSO arrays. The dimension of the crystal matrix elements are $1.4 \times 1.4 \mathrm{~mm}^{2}$ in cross section and $12 \mathrm{~mm}$ in depth. The VrPET detector modules are positioned in opposite sides of a $140 \mathrm{~mm}$ diameter ring, providing a transaxial field of view of $86.6 \mathrm{~mm}$ diameter and an axial field of view of $45.6 \mathrm{~mm}$. The experimental results obtained in the performance tests are transaxial resolution of $1.5 \mathrm{~mm}$ FWHM in the CFOV, and the axial resolution of 2.3 $\mathrm{mm}$ FWHM. The absolute coincidence sensitivity is $2.22 \%$ for a coincidence window of $6 \mathrm{~ns}(100-700 \mathrm{keV})$. The imaging capability of the PET unit is demonstrated on phantom and animal studies.
\end{abstract}

Index Terms- Positron emission tomography (PET), small animal imaging, $\mathrm{X}$-ray computed tomography (CT).

\section{INTRODUCTION}

$\mathrm{T}$ HE increasing number of animal models of human diseases and the radiolabeling of small molecules, has made small animal PET a valuable tool in medical research. Actual tendency in clinical scanners is to combine the functional information of PET images with an anatomical imaging modality such as X-ray CT, whose fusion improves the image interpretation. Given the increasing importance of the use of mice in biomedical research, this same tendency is currently observed in small-animal dedicated tomographs, and several multi-modality systems are being developed and introduced in the market [1].

Manuscript received November 14, 2008.

This work is partially funded by the CD-TEAM project, CENIT Program, Spanish Ministerio de Industria and with grants from the Ministerio de Educación y Ciencia, Projects TEC2007-64731 and TEC2008-06715-C02-01.

E. Lage, J. J. Vaquero, A. Sisniega, G. Tapias, V. Garcia, A. RodriguezRuano and M Desco are with the Unidad de Medicina y Cirugia Experimental, Hospital General Universitario Gregorio Marañon, Madrid, Spain. (E. Lage email: elage@hggm.es, J.J.Vaquero e-mail: juanjo@mce.hggm.es).

S. España is with the Departamento de Física Atómica, Molecular y Nuclear. Facultad de Ciencias Físicas, Universidad Complutense de Madrid. Spain.

A. Udias is with the Departamento de Estadistica e Investigación Operativa, Universidad Rey Juan Carlos, Fuenlabrada, Spain.
A difficulty for the widespread use of small-animal PET/CT is the high investment involved in installing and maintaining tomographs. The most expensive components of PET subsystems are by far the detection elements, which are composed by processing electronics and gamma-ray sensors usually arranged in axial rings around the sample in order to provide a high sensitivity and a large FOV.

The VrPET/CT system, implemented using only two opposed detector blocks mounted on a rotary gantry, makes use of a custom hardware/software design, specifically intended to provide high quality images and adequate temporal resolution. The PET component is based on an improved version of the rPET scanner (Suinsa Medical Systems, Spain) [2] and a recently developed cone-beam micro-CT system, whose design and performance evaluation has already been detailed in other publications [3]-[5]. This paper focuses on the PET subsystem design and evaluation as well as on the procedures required to obtain high quality images.

\section{SYSTEM CONSTRUCTION}

\section{A. PET Detector Modules}

The system uses two double-detector modules. Each module is composed of two single detectors based on a $30 \mathrm{x}$ 30 lutetium-yttrium oxyorthosilicate (LYSO) crystal array coupled to a Hamamatsu H8500 Position Sensitive Photomultiplier (PS-PMT) through optical grease BC-630 (Bicron/Saint Gobain). The scintillator matrices were arranged using individual crystals with all his facets mechanically polished and separated by a 100 -micron thick lumirror reflector layer.

The detectors are mounted using black delrim enclosures (Fig. 1) which fit in a V-shaped shielded box that attaches the detectors to the gantry. The flat panel PS-PMTs has 12 stages of metal channel dynode and $8 \times 8$ multiple anodes which provide an active area of $49.0 \mathrm{~mm}$ square. The 64 anode signals of the PMT are combined into 4 position signals, by a readout circuit based on passive elements, which are amplified to adapt them to the dynamic range of the data acquisition system. These electronic components together with an additional stage for the last dynode signal of the PMT and a high voltage supply are included in a compact PCB stack, directly attached to the PMT sockets (Fig. 1). 


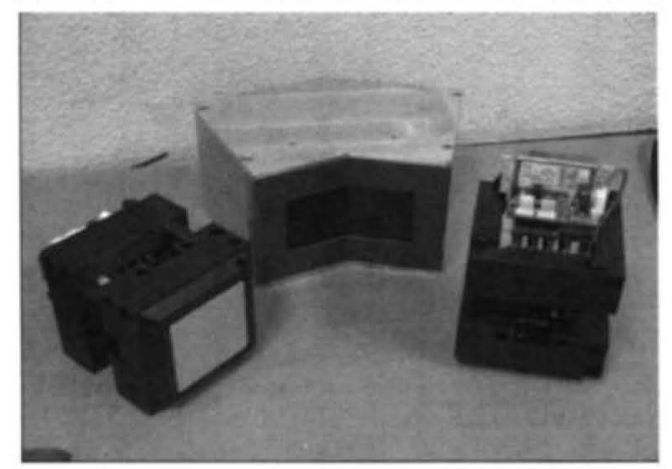

Fig 1. VrPET detector modules. Each detector module contains two basic detection units packed in black delrim enclosures which fit in a light tight lead $(\mathrm{Pb})$ shielded box that doubles as EM screen

\section{B. Gantry Geometry and Acquisition Protocols}

The detector modules are attached to a rotary gantry arranged in two V-shaped blocks. The small-animal CT scanner has been assembled in such a way that the centers of rotation for both modalities are intrinsically aligned (Fig. 2). Opposed PET detector heads are positioned at $140 \mathrm{~mm}$ centerto-center distance (Fig. 2) to form 30 virtual detector partial rings with a $1.5 \mathrm{~mm}$ pitch. The angle in the transaxial plane between detectors of the same block $\left(143.5^{\circ}\right)$ was selected to provide a Field of View (FOV) slightly larger than that of the CT system $(\sim 75 \mathrm{~mm} \times 75 \mathrm{~mm} \times 75 \mathrm{~mm})$, while allowing the $\mathrm{X}$-ray cone-beam to illuminate the $\mathrm{X}$-ray detector surface without obstacles. The resulting FOV of the PET subsystem is $86.6 \mathrm{~mm}$ diameter in the transaxial dimension and $46.5 \mathrm{~mm}$ in axial (dimension of the crystal array). The scanner also includes a linear motion stage to move the sample along the axial FOV for whole body studies. Both motion axes (rotating gantry and bed) have submillimeter resolution and are controlled by means of stepper motors and digital drives using a general purpose personal computer, which also manages the different system elements during PET or CT data acquisitions.

Although the suitability of similar configurations has been demonstrated for simultaneous metabolic and anatomic imaging of small animals [6], the VrPET/CT is intended to acquire these modalities sequentially. This design specification was derived from the fact that the most commonly used CT data acquisition protocol, intended to provide anatomical templates for the PET images, is time inexpensive (about 1.5 minutes per bed position).

\section{Acquisition Electronics}

Last dynode signals of the four PET detectors are fed into a pulse discriminator circuit through coaxial cables. This circuit generates timing signals which allows detecting coincident photons occurred within a configurable coincidence window $(3,5$ or $10 \mathrm{~ns})$. As a result of coincidence detection in any pair of detectors, trigger signals are generated to start the digitization of the position signals necessary to characterize the event.

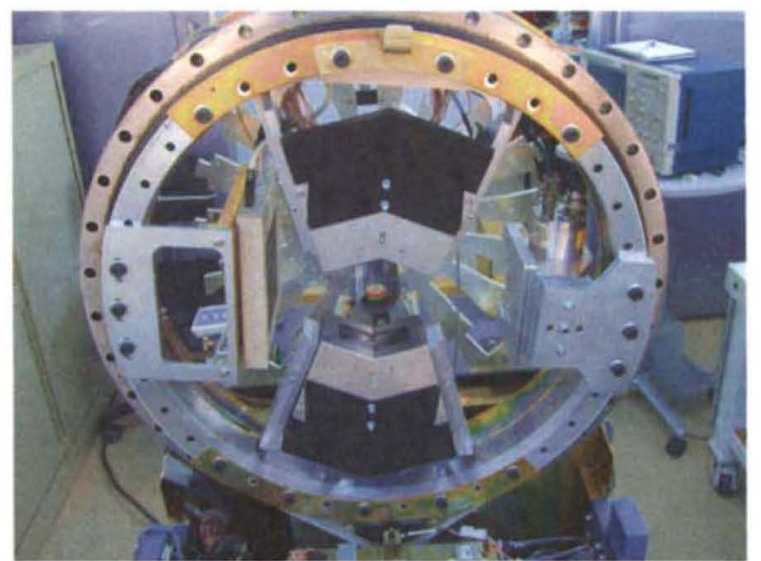

Fig 2. VrPET/CT (SUINSA Medical Systems) gantry implementation. The machine is based on a rotary gantry in which four PET detectors arranged into $2 \mathrm{~V}$-shaped blocks and a small-animal CT scanner composed by a flat panel detector (left) and a micro-focus $X$-ray tube (right); the centre of rotation for both modalities is intrinsically aligned.

These position signals ( $\mathrm{X}+, \mathrm{X}-, \mathrm{Y}+$, and $\mathrm{Y}-)$ are integrated for a $160 \mathrm{~ns}$ period and digitized using 12-bit ADCs. In this way, coincidences are discriminated via hardware and sent to the PC in order of arrival, and the system does not acquire in single mode nor includes timing information in the data events. Events from analog-to-digital converters are sent to a communications module that packs frames and feeds them to an acquisition PC via a Gigabit Ethernet interface. The data acquisition system also contains a counter module with seven general purpose scalers. The current value of these registers is included on the data frame for each coincidence detected, providing synchronization information such as the detectors position, time or gating signals.

\section{Data Processing and Reconstruction}

For all the reconstruction modes, list data are binned into sinograms consisting on 117 rays and 190 angles for each of the $30^{2}$ possible crystal combinations. During this process corrections such as decay, normalization and dead time are applied to the sinogram. The normalization correction is based on a measurement of an annulus shaped cylinder, homogeneously filled with an aqueous solution containing ${ }^{18} \mathrm{~F}$ fluoro-deoxyglucose (FDG). A normalization 3D-sinogram is derived from this measurement and used to correct the data sinograms before applying analytic reconstruction protocols.

For $2 \mathrm{D}$ reconstructions, the $3 \mathrm{D}$ sinograms are converted to 59 slices by Fourier rebinning (FORE) or single slicerebinning (SSRB) algorithms with a configurable maximum ring difference. $2 \mathrm{D}$ sinograms are reconstructed with standard 2D filtered-backprojection (FBP) or a fast iterative 2D OSEM algorithm [7]-[8]. A 3D-OSEM reconstruction algorithm has also been implemented [9].

\section{Performance Evaluation}

Evaluation of the system performance was carried out according to the NEMA NU 4-2008 standard [10]. The 
experiments described below were performed with energy windows of 100-700, 400-700 or $250-650 \mathrm{keV}$, and with a 6 ns coincidence window width.

\section{A. Spatial Resolution}

The spatial resolution of the system was assessed with an encapsulated ${ }^{22} \mathrm{Na}$ point source of $0.3 \mathrm{~mm}$ diameter. Different measurements were taken in the central slice of the axial FOV at several radial distances from the geometrical center $(0,5 \mathrm{~cm}$ increments). Resolution at the same transaxial points was also evaluated at $1 / 4$ axial FOV without noticeable variations. The scan period was 5 minutes at each position to collect more than $100 \mathrm{k}$ counts. The list mode were rebinned into 2D sinograms by SSRB and reconstructed by FBP with a ramp filter.

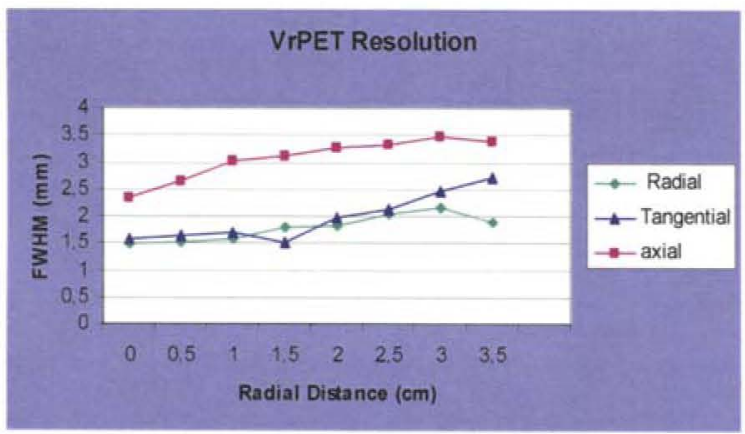

Fig 3. Spatial resolution measured from reconstructed image

The spatial resolution was calculated as the full width at half maximum (FWHM) of the profiles in the radial, axial and tangential directions. Fig. 3 shows the spatial resolution (FWHM) as a function of the radial distance from the center. The transaxial resolution at the CFOV is roughly $1.5 \mathrm{~mm}$ and the axial resolution is $2.34 \mathrm{~mm}$.

\section{B. Sensitivity}

System sensitivity was evaluated using the same source as in the previous test, carefully centered in axial and transaxial directions. Two-minute acquisitions were obtained as the source was being stepped in $0.5 \mathrm{~mm}$ steps across the scanner from end to end of the axial FOV. The activity was low enough so that the dead time loss was negligible. The result of the absolute sensitivity of the scanner along the axial FOV is presented in fig 4 . The peak sensitivity at the CFOV is $2.22 \%$ with a $100-700 \mathrm{keV}$ energy window, $1.56 \%$ with a $250-650$ $\mathrm{keV}$ energy window and $0.94 \%$ for the $400-700 \mathrm{keV}$ energy window.

\section{Scatter Fraction and Noise Equivalent Count Rate}

A mouse-sized NEMA test phantom was located on the animal bed in such a way that the line source was positioned nearest to the subject bed, with the phantom centered in the transverse an axial fields-of-view. The initial activity concentration inside the $\mathrm{FOV}$ was $24.8 \mu \mathrm{Ci} / \mathrm{cc}$, the data were acquired for 12 hours in 20 min frames and sinograms were generated for each acquisition frame and for each slice by single-slice rebinning. The coincidence background noise due to the intrinsic radioactivity contained in LYSO crystals was not subtracted from the measured coincidences since the coincidence background noise in this machine is less than 20 cps.

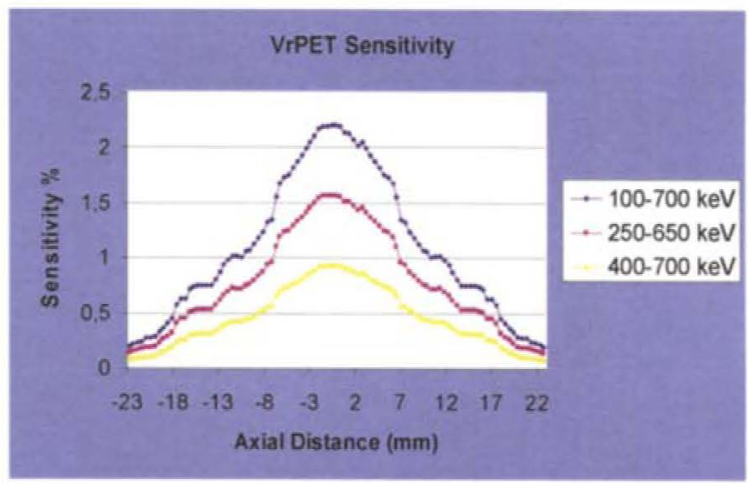

Fig 4. Sensitivity of the VrPET scanner. The slice absolute sensitivity is plotted as a function of the coordinate along the axial axis of the scanner.

The scatter fraction (SF) was analyzed using the last five frames. These frames contained random event rates and count loss rates below $1.0 \%$ of the true event rate. For these acquisitions and due to the low activity, it is assumed that the number of random counts is negligible and the difference between true and prompt count rates is only due to scattered events. The average SF was $29.1 \%$ for this phantom.

The noise equivalent count rate (NEC rate) was calculated from the true and prompt coincidences, as defined in the NEMA protocol for this kind of scanner. Fig 5 shows prompt count rate, true count rate, NEC rate and random plus scatter count rate curves. The peak count rate of the NEC was 50.95 $\mathrm{kcps}$ at $19.31 \mathrm{uCi} / \mathrm{cc}$ for this phantom.

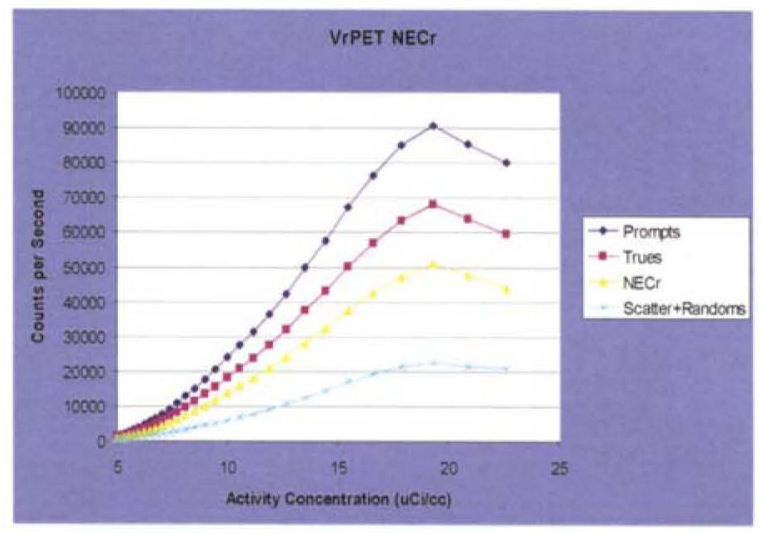

Fig 5. Count rate capability of the VrPET system as a function of the line source activity divided by the total volume of the cylindrical mouse-sized phantom.

\section{Imaging Capabilities}

To demonstrate the imaging capabilities of the VrPET scanner several studies were carried out, including in-vivo studies with rats and mice. Fig 6 shows a transaxial slice of a Derenzo phantom, integrated over 20 slices. The data were 
acquired for 30 minutes in a single bed position; the total activity in the phantom at acquisition start was $142 \mu \mathrm{Ci}$.

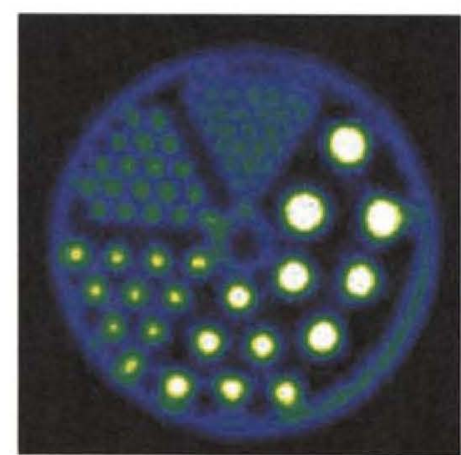

Fig 6. Derenzo Phantom transaxial image. After analytic image reconstruction (SSRB+2D FBP), 20 slices were integrated. The smallest rods $(1.2 \mathrm{~mm})$ are resolved

Fig 7 shows maximum intensity projection (MIP) views of a $275 \mathrm{~g}$ Wistar rat (left) and a $37 \mathrm{~g} \mathrm{BALB} / \mathrm{c}$ mouse (right). The rat was injected with $1.62 \mathrm{mCi}$ of FDG and the mouse with $430 \mathrm{uCi}$ of the same radiopharmaceutical. The measurements in both cases started 60 minutes after injection and data were acquired for 20 minutes for each bed position ( 3 positions in both cases). The images were reconstructed with 3D-OSEM, where no attenuation and scatter corrections were employed.

\section{CONCLUSIONS}

A rotary PET/CT scanner has been developed and its initial performance was evaluated. This work demonstrates the feasibility of a coplanar PET/CT system for in-vivo imaging of small laboratory animals. The physical configuration of this system provides intrinsically co-registered datasets, thus not being necessary to reposition the animal to perform multi modality imaging.

Although there is still some room for improvement, such as the acquisition dead time, the performance values obtained using this configuration makes this scanner suitable for most of the PET applications, while the cost derived from detectors and electronics is significantly reduced as compared with a full-ring configuration.

\section{ACKNOWLEDGMENT}

The authors thanks SUINSA Medical System Engineering department for their support and the development of the mechanical parts.
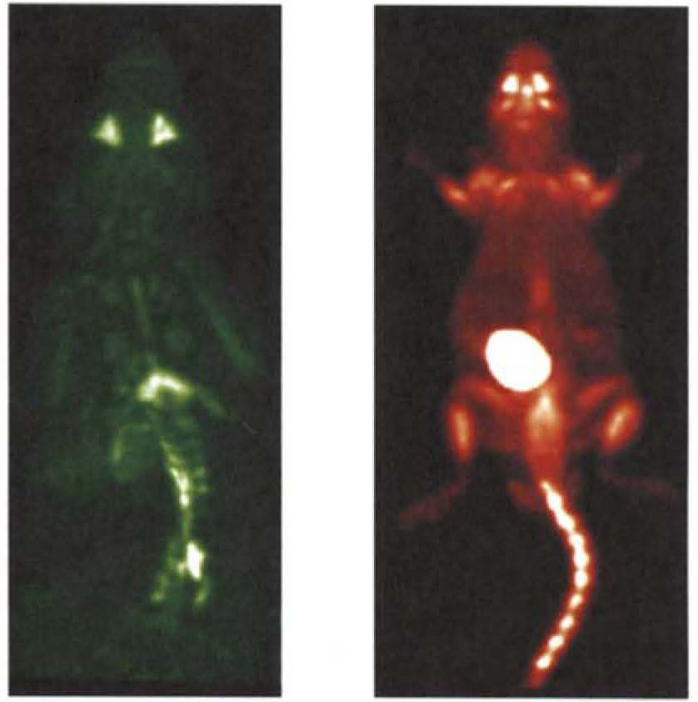

Fig 7. FDG images of rat (left) and mouse (right) acquired with the VrPET. Both images were reconstructed using 3D-OSEM. Total acquisition time was 1 hour in both cases.

\section{REFERENCES}

[1] H Liang, Y Yang, K Yang, Y Wu, J M Boone and Simon Cherry. "A microPET/CT system for in vivo small animal imaging", Physycs in Medicine an Biology. 52 (2007) 2881-3894.

[2] Suinsa S.A. C/ Primavera 39. Torrejón de Ardoz, 28850, Madrid, Spain. http://www. suinsa.com/

[3] E Lage, J J Vaquero, S Redondo, M Abella, G Tapias, A udias, M Desco. "Design and Development of a High performace Micro-CT System for Small-Animal Imaging", 2006 NSS / MIC Conference Record 3549-3552.

[4] S Redondo, J I Vaquero, E Lage, M Abella, G Tapias, A udias, M Desco. "Assessment of a New CT System for Small Animals", 2006 NSS / MIC Conference Record 3553-3556

[5] JJ Vaquero, S Redondo, E Lage, M Abella, A Sisniega, G Tapias, ML Soto-Montenegro, M Desco. "Assessment of a New High-Performance Small-Animal X-Ray Tomograph". IEEE T Nucl Sci, 55(3): 898-905, 2008

[6] Andrew L Goertzen et al "Simultaneous molecular and anatomical imaging of the mouse in vivo". 2002 Phys. Med. Biol $474315-4328$

[7] J. E. Ortuño, P. Guerra, J. L. Rubio, G. Kontaxakis, and A. Santos, "3I)OSEM iterative image reconstruction for high-resolution PET' using precalculated system matrix," Nuclear Instruments \& Methods in Physics Research Section a-Accelerators Spectrometers Detectors and Associated Equipment, vol. 569, (2), pp. 440-444, 2006

[8] J. E. Ortuño, J. L. Rubio, P. Guerra, G. Kontaxakis, M. Desco, J. J. Vaquero and A. Santos "Efficient Methodology for 3D Statistical Reconstruction of High Resolution Coplanar PET/CT Scanner", IEEE MIC 08 Conference Record, in press.

[9] JL Herráiz, S España, JJ Vaquero, M Desco, JM Udias. "FIRST: Fast Iterative Reconstruction Software for (PET) Tomography". Phys Med Biol, 51(18): 4547-4565, 2006

[10] National Electrical Manufacturers Association. Performance Measurements for Small Animal Positron Emission Tomographs (PETs). Rosslyn, VA; 2008. Standars Publication NU $4-2008$ http://www.nema org/stds/nu4.cfm 